\title{
Coverage Problems in Wireless Sensor Networks: Designs and Analysis
}

\author{
My T. Thai Feng Wang Ding-Zhu Du \\ Dept. of Computer Science \& Enginering \\ University of Minnesota \\ Minneapolis, MN 55455 \\ \{mythai, fwang, dzd\}@cs.umn.edu
}

\begin{abstract}
Recently, a concept of wireless sensor networks has attracted much attention due to its widerange of potential applications. Wireless sensor networks also pose a number of challenging optimization problems. One of the fundamental problems in sensor networks is the coverage problem, which reflects the quality of service that can be provided by a particular sensor network. The coverage concept is defined from several points of view due to a variety of sensors and a wide-range of their applications. Several different designs and formulations of coverage problems have been proposed. They are subject to various topics such as types of interest regions (areas vs. targets) and different objectives (maximum network lifetime, minimum coverage breach) with other constraints. In this paper, we survey the state-of-the-art coverage formulations, present an overview and analysis of the solutions proposed in recent research literature.
\end{abstract}

Keywords: wireless sensor networks, energy efficiency, sensor scheduling, coverage.

\section{Introduction}

Recent advances in technology have made possible the creation of wireless sensor networks. Wireless sensor networks can be used in a widerange of potential applications such as national security, surveillance, health care, biological de- tection, environmental monitoring, and many other applications $[1,2,3]$. A wireless sensor network consists of a large number of tiny sensor nodes to accomplish a large sensing task. Sensor nodes are small devices equipped with one or more sensors, one or more transceivers, processing, storage resources and possible actuators [4]. Sensors in a network can cooperatively gather information from an interest region of observation and transmit this collected information to a base station. There are two types of data sent to the base station: 1) event-drive and 2) on-demand. In the former case, the data is sent to the base station when one or more sensors detect an event in the vicinity. In the latter case, the data is sent from the sensors to the base station based on an explicit request.

Energy efficiency is one of the most critical issues in wireless sensor networks. With the current available technology, sensors are battery powered and have a limited weight. These characteristics globally affect the application lifetime. Thus prolonging network lifetime is an important part of the system design in wireless sensor networks. One of the most prominent methods is based on the scheduling sensor activity so that a set of active sensors can handle a required task while the rest of the redundant sensors can enter into sleep mode. In [6], the authors pointed out that the energy consumption in the sleep state is about 15 times less than that in the active state. In addition, the lifetime of a battery discharging in short bursts with significant off-time 
is approximately twice as much as that in a continuous mode of operation [5]. These evidences clearly show that the scheduling sensor activity can efficiently extend network lifetime.

An important problem in wireless sensor networks is the coverage problem. The goal is to have each location in the physical space of interest within the sensing range of at least one sensor. In wireless sensor networks, sensors are densely deployed. The number of sensors deployed is usually higher than optimum required due to the lack of precise sensor placement, especially when the interest region is inaccessible. Thus it is possible to turn some sensors off while guaranteeing the complete coverage of the interest region.

In this paper, we survey the state-of-the-art coverage formulations, present an overview and analysis of the solutions proposed in recent research literature. One of the main objectives of the coverage problem is to prolong network lifetime. Besides this objective, the coverage problem has many variants based on the other constraints of networks and objects to be covered. Based on the objects to be covered, there are two different types: 1) Area Coverage and 2) Target Coverage. As the names reflect themselves, the interest region to be covered in the former is an area while in the latter is a set of targets. Here, a target can be a point. Indeed, an area coverage can be transformed to a target coverage, which we discuss further in section 3. Many algorithms, including centralized, distributed and localized, have been proposed [13]-[20]. Most of these algorithms focus on a mechanism to organize the sensors into a number of subsets such that each subset can completely cover an interest region in order to extend network lifetime.

Other network constraints that the coverage algorithms need to take into consideration are: 1) Connectivity, 2) Bandwidth Limitation, and 3) Fault Tolerance. We briefly introduce these variants as follows.

Connectivity is another important problem. The active nodes must form a connectivity so that the sensed data can be sent to a base sta- tion. Thus in this survey, we also focus on the connected coverage problem. We will present a simple yet important relationship between coverage and connectivity in wireless sensor networks [11]. We then discuss and analyze a few important work $[11,12]$ in this area.

Due to the resource limitation of sensor nodes, another fact that we need to take into consideration is the bandwidth limitation. When organizing sensors into a number of subsets, the number of available channels must be enough for the active sensors to communicate. Otherwise, some sensors cannot send data back to base stations. Thus the number of sensors in each subset must be less than or equal to the number of available channels. With this constraint, coverage breach can occur, i.e., some targets are not covered. For a good design, the coverage breach should be minimize, which is a minimum breach coverage problem. In this paper, we present a formal definition of the minimum breach coverage problem and discuss the recent papers $[9,10]$.

In addition, fault tolerance is an important issue in wireless sensor networks. For the coverage problem, one must ensure that the interest region is under monitored all time although some sensor nodes may fail. For each point of the interest region, there must be at least $k$ sensors cover it. This is a $k$-coverage problem, which is also addressed in this paper. Similar to the coverage problem, the connectivity of the $k$-coverage problem also need to study, which is a connected $k$-coverage problem. There are not many work on the $k$-coverage problem in literature. We here discuss some algorithms proposed recently and expect to see more work in this specific area $[21,22]$.

The rest of this paper is organized as follows. Section 2 presents the preliminaries and the problem definitions about different variants of the coverage problem. Section 3 discusses and analyzes the recent proposed algorithms with an objective of maximizing network lifetime. The $k$ coverage and connected coverage and connected $k$-coverage problems are presented in section 4 and 5 respectively. The minimum coverage 
breach problem is discussed in section 6 . Finally, section 7 concludes this paper with discussion and outlooks.

\section{Preliminaries and Problem Definitions}

In this paper, we consider a sensor network consisting of $n$ sensors $s_{i}, i=1 \ldots n$. A sensor's radio can be in one of the four following states: transmit, receive, idle, or sleep. The sleep state is when the radio is off and the idle state is when the transceiver is neither transmitting nor receiving. Each sensor $s_{i}$ can be active continuously for a maximum of $b_{i}$ unit time. $n$ sensors are deployed to monitor an interest region which is either an area or a set of targets. Let $r_{i}$ and $R_{i}$ denote the sensing range and the communication range of a sensor $s_{i}$ respectively. Note that we assume a sensing area of a node $s_{i}$ being a disk centered at sensor $s_{i}$ with radius $r_{i}$. Likewise for the communication range. A sensor $s_{i}$ covers a target $q$ if and only if target $q$ is in the sensing disk of $s_{i}$. Formally, let consider a target as a point, we can define:

Definition 1 Sensor Covering a Point: A sensor $s_{i}$ is said to cover a point $q$ if and only if the distance $d\left(q, s_{i}\right) \leq r_{i}$

The distance function $d$ can be the Euclidean distance function. Likewise, the Sensor Covering an Area can be defined as follows:

Definition 2 Sensor Covering an Area: A sensor $s_{i}$ is said to cover an area $A$ if and only if for every point $q$ in $A$, the distance $d\left(q, s_{i}\right) \leq r_{i}$.

Two sensors can communicate directly with each other if and only if each sensor is inside the communication range of the other. Formally, we have:

Definition 3 Direct Communication: Sensor $s_{i}$ and $s_{j}$ can communicate directly with each other if and only if $d\left(s_{i}, s_{j}\right) \leq r_{j}$ and $d\left(s_{j}, s_{i}\right) \leq$ $r_{i}$

\section{Definition 4 Communication}

Graph:

Given a sensor network consisting of $n$ sensors, the communication graph of the network is an undirected graph $G_{C}=\left(V, E_{C}\right)$ where $V$ is a set of sensors and $E_{C}$ is a set of edges consisting edges between any two sensors that can communicate directly with each other.

The communication subgraph induced by a set of active sensor $S$ is the subgraph of $G_{C}$ after removing the inactive sensors (sensors are not in $S$ ) and their associated edges. We are now ready to introduce the four main problem definitions that are covered in this paper.

Definition 5 Maximum Lifetime Coverage: Given a network consisting of $n$ sensors and an interest region, find a family of ordered pairs $\left(S_{j}, t_{j}\right), j=1 \ldots p$, where $S_{j}$ is a set of sensors that completely cover the interest region and $t_{j}$ is the time duration for $S_{j}$ to be active, such that to maximize $t_{1}+\ldots+t_{p}$. For each sensor $s_{i}$, $s_{i}$ appears in $S_{1}, \ldots, S_{p}$ with a total time at most $b_{i}$.

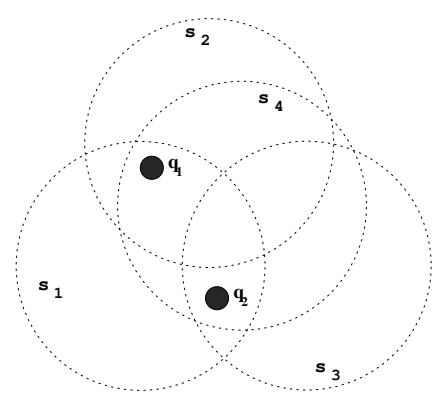

Figure 1: An Example of Target Coverage

In this definition, the interest region can be either an area or a set of targets. If the interest region is an area, the maximum lifetime coverage problem is an area coverage problem with an objective of maximizing network lifetime. Likewise, if the interest region is a set of targets, the maximum lifetime coverage problem is a target coverage problem with an objective of maximizing network lifetime. Note that after finding a family of ordered pairs $\left(S_{j}, t_{j}\right)$ as defined in definition 
5 , each set $S_{j}$ will be active successively to completely cover the interest region. For example, Figure 1 shows a network consisting of four sensor $s_{1}, s_{2}, s_{3}, s_{4}$ and 2 targets $q_{1}, q_{2}$. Assume that $b_{1}=\ldots=b_{4}=1$, we can find the family of ordered pairs $\left\{\left(\left\{s_{1}\right\}, 1\right),\left(\left\{s_{2}, s_{3}\right\}, 1\right),\left(\left\{s_{4}\right\}, 1\right)\right\}$. So at time instance 1 , sensor $s_{1}$ is active and the rest of the other sensors are in the sleep mode. After 1 unit time, sensor $s_{1}$ goes to sleep and sensors $s_{2}, s_{3}$ are active to monitor targets $q_{1}, q_{2}$. These two sensors are active for 1 unit time. Sensor $s_{4}$ then stays up while both sensors $s_{2}$ and $s_{3}$ go back to sleep. With this strategy, the network lifetime is 3 instead of 1 if all sensors are active.

\section{Definition 6 Connected Coverage Prob-}

lem: Given a sensor network consisting of $n$ sensors and an interest region, find a family of ordered pairs $\left(S_{j}, t_{j}\right)$ satisfying the following two conditions:

1. Satisfy the conditions of the Maximum Lifetime Coverage problem

2. The communication graph induced by $S_{j}$ is connected

Definition 7 K-Coverage Problem: Given a sensor network consisting of $n$ sensors and an interest region A, find a family of ordered pairs $\left(S_{j}, t_{j}\right)$ satisfying the following two conditions:

1. Satisfy the conditions of the Maximum Lifetime Coverage

2. Each point $q$ in $A$ is covered by at least $k$ distinct sensors in each $S_{j}$

Definition 8 Connected K-Coverage Problem: Given a sensor network consisting of $n$ sensors and an interest region, find a family of ordered pairs $\left(S_{j}, t_{j}\right)$ satisfying the following two conditions:

1. Satisfy the conditions of the K-Coverage problem

2. The communication graph induced by $S_{j}$ is connected
In case of limited bandwidth as mentioned in the previous section, in a design of the coverage algorithms, we must consider a number of available channels. Bandwidth is defined as either the total number of time slots if a time division scheme is used on a single shared channel, or the total number of channels if multiple channels are available. Under bandwidth constraints, if there are $W$ available channels, for example, and there are more than $W$ sensors in a subset $S_{i}$, then obviously some sensors cannot access the channels for data transmission. Hence, the size of each subset $S_{i}$ must be less than or equal $W$. With this constraint, coverage breach may occur, i.e., some targets are not covered. Researchers hence address this problem as a minimum coverage breach problem as follows.

Definition 9 Minimum Coverage Breach: Given a sensor network consisting of $n$ sensors and an interest region, find a family of ordered pairs $\left(S_{j}, t_{j}\right)$ such that the total coverage breach is minimum.

Some work in literature may define all the above problems slightly different depending on their methods and designs. In this case, we address the differences in detail when discussing each specific work. Otherwise, these definitions are used through out the paper without mention. We end this section with the following theorem:

Theorem 1 The Maximum Lifetime Coverage, the Connected Coverage problem, the $k$-Coverage problem, the Connected $k$-Coverage problem, and the Minimum Coverage Breach are NP-complete

\section{Maximum Lifetime Coverage}

In this section, we first present the relationship between the area coverage and target coverage problems, the present and analyze the recent proposals for both problems. The algorithms are divided into two categories: 1) Centralized and 2) Distributed and Localized. 


\subsection{Area and Target Coverage Rela- tionship}

From the Sensor Covering a Point and Sensor Covering an Area definitions, one can easily see that there must exist an relationship between the area coverage problem and the target coverage problem. Indeed, the area coverage problem can transform to the target coverage problem $[17,13]$.

Definition 10 Field: A field is a set of all points such that they are covered by the same set of sensors.

Consider an example in Figure 2(a), the circles are the sensing disks of sensors. The rectangle is an interest region. According to the above definition, there are 8 fields in Figure 2(a). Let targets $q_{1}, \ldots, q_{8}$ represent these 8 fields, then the area coverage problem can transform to the target coverage problem as shown in Figure 2(b). The authors in [13] proposed an efficient way

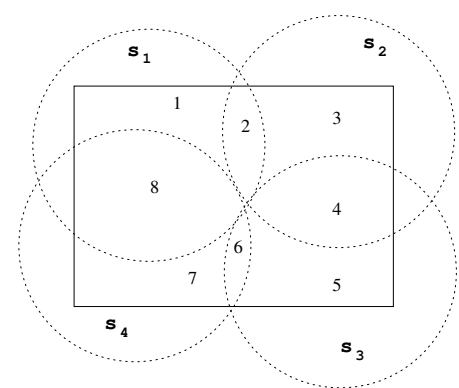

(a) An Area Coverage Problem

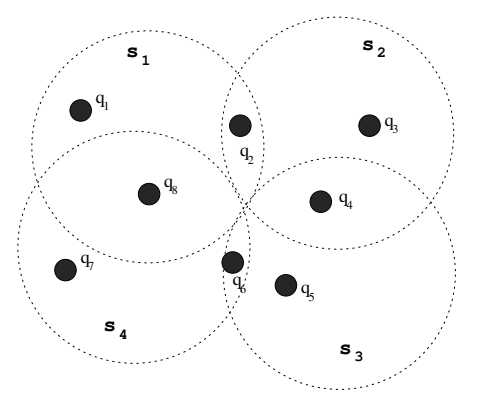

(b) Equivalent Target Coverage

Figure 2: Area and Target Coverage Relationship

to compute all these fields without considering all the points. In particular, let a planar graph $P=(V, E)$ represent an interest region where $V$ is a set of intersections of boundaries of sensing disks and $E$ is a set of edges such that an edge $u v \in E$ if and only if $u$ and $v$ are adjacent intersection points along the boundaries of sensing disks. Then the faces of the planar graph $P$ discretize the interest region, rather than using a set of grid points as proposed in [17].

\subsection{Centralized Algorithms}

Many centralized algorithms have been proposed. Most of them use the scheduling mechanism, that is to find a family of ordered pairs $\left(S_{j}, t_{j}\right)$ so that each set can be active successively. The resultant subsets $S_{j}$ may be disjoint or non-disjoint depending on different algorithms. In addition, instead of maximizing the total network lifetime, some work focused on finding a subset $S_{j}$ with a minimum size.

The authors in [17] modeled the area coverage problem as the maximum number of disjoint sets problem. First, divide a given interest region (an area $A$ ) into a set of fields. After the fields are recognized, an heuristic called Most Constrained-Minimally Constraining Covering computes the maximum number of disjoint sets such that each set can completely monitors all fields. Since these sets are disjoint, the more disjoint sets, the longer network lifetime. These disjoint sets are constructed successively. The basic idea of this heuristic is to minimize the coverage of sparsely covered fields within one set cover. To do so, a critical element need to be identified.

Definition 11 Critical Element: A critical element is a field covered by a minimal number of sensors.

After identifying the critical element, a cover set is constructed by selecting sensors that cover the critical element by following priority:

1. cover a high number of uncovered fields

2. cover sparsely covered fields 


\section{3. do not cover fields redundantly}

The time complexity of this heuristic is $O\left(n^{2}\right)$. Also, in this study, the authors assumed that the of all sensors is are equal, i.e., $b_{i}=b$. After constructing all disjoint dominating sets, each set is active for $b$ unit time successively.

Using a similar approach, finding a maximum number of disjoint set, Cardei et al. proposed another algorithm for the area coverage problem. The authors modeled the coverage problem as the maximum number of disjoint dominating sets in an undirected graph $G=(V, E)$ where $V$ is a set of sensors and $E$ is a set of edges such that $u v \in E$ if and only if $u$ and $v$ are each within other's sensing range. The authors proved that the maximum number of disjoint dominating sets is NP-complete. A graph-coloring mechanism was proposed to compute the maximum number of disjoint dominating sets. This approximation algorithm has two phases. At the first phase, all vertices are colored using the sequential coloring algorithm, i.e., coloring vertices one by one using a least possible color not appearing in its neighbors. At the second phase, after coloring all vertices, an $O\left(n^{3}\right)$ heuristic is employed to construct the disjoint dominating sets. However, note that the dominating set does not guarantee the complete coverage. This is a tradeoff to prolong network lifetime. A simulation results show that the number of sets obtained from [18] is 1.5 to 2 times more than that of [17].

Later, Cardei and Du [16] proposed another method to efficiently compute the maximum number of disjoint set covers where each set completely covers all the targets. Here, the authors used the target model to study the coverage problem. The maximum number of disjoint set covers problem was reduced to a maximum flow problem, which is then formulated as a mixed integer programming. The simulation results show that the number of sets obtained from [16] is slightly better than that of [17].

The above algorithms focus on finding a maximum number of disjoint sets. However, the authors in [13] and [15] independently found that organizing sensors into a maximum number of

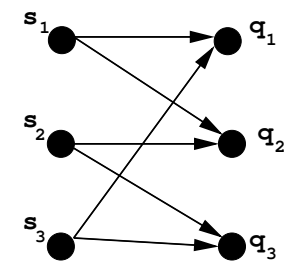

Figure 3: An Example for non-disjoint Sets

non-disjoint set covers may obtain a longer network lifetime. For example, in Figure 3, the network consists of 3 sensors $s_{1}, s_{2}$, and $s_{3}$ that cover 3 targets $q_{1}, q_{2}$, and $q_{3}$. Target $q_{1}$ is covered by sensors $s_{1}$ and $s_{2}$. Target $q_{2}$ is covered by sensors $s_{2}$ and $s_{3}$. Target $q_{3}$ is covered by sensors $s_{3}$ and $s_{1}$. Let us assume that $b_{1}=b_{2}=b_{3}=1$, if we organize sensors into disjoint set covers, i.e., $S_{1}=\left\{s_{1}, s_{2}\right\}$, the network lifetime is 1 unit time. If we organize sensors into non-disjoint set covers, i.e., $S_{1}=\left\{s_{1}, s_{2}\right\}, S_{2}=$ $\left\{s_{2}, s_{3}\right\}, S_{3}=\left\{s_{1}, s_{3}\right\}$, where each set is active for 0.5 unit time, then the network lifetime is 1.5 unit time. Based on this observation, the authors in [13] and [15] proposed two different algorithms using the Linear Programming techniques. The main approach is to find a family of ordered pairs $\left(S_{j}, t_{j}\right)$ so that the total lifetime is maximum. Each set $S_{j}$ completely covers all the targets. And the sensor $s_{i}$ appears in all $S_{j}$ sets at most $b_{i}$ unit time.

In [13], Berman et al. introduced a new approach for the area coverage problem using a packing Linear Programming technique. In this approach, again, sensors are divided into nondisjoint sets. First, all fields of the given area are identified using the planar graph approach as mentioned in the previous section. Let $p$ denote the number of set covers, the authors formulated this coverage problem as follows:

$$
\begin{array}{ll}
\operatorname{maximize} & \sum_{j=1}^{p} t_{j} \\
\text { subject to } & \sum_{j=1}^{p} C_{i j} t_{j} \leq b_{i} \\
\text { where } & C_{i j}=1 \text { iff } s_{i} \in S_{j} \\
& C_{i k}=0 \text { otherwise }
\end{array}
$$

Here, $C_{i j}$ is a matrix with rows $i=1 \ldots n$ representing each sensor and columns $j=1 \ldots p$ repre- 
senting each set cover.

Based on the $(1+\epsilon)$-approximation GargKonemann algorithm [7], the authors in [13] proposed an approximation algorithm to solve the above formulation, that is, to find the value $C_{i j}$ and $t_{j}$ so that $\sum_{j=1}^{p} t_{j}$ is maximum. The authors also proved that the performance ratio of their proposal is within a factor of $(1+\epsilon)(1+l \ln n)$ for any $\epsilon>0$.

The authors in [15] proposed another model called Maximal Set Covers (MSC) to solve the target coverage problem. This problem is formulated as follows:

$$
\begin{array}{ll}
\text { maximize } & \sum_{j=1}^{p} t_{j} \\
\text { subject to } & \sum_{j=1}^{p} x_{i j} t_{j} \leq 1 \forall i \\
& \sum_{i \in C_{k}} x_{i j} \geq 1 \forall k, j \\
\text { where } & x_{i j}=0,1\left(x_{i j}=1 \text { iff } s_{i} \in S_{j}\right)
\end{array}
$$

Here, $C_{k}$ denote a set of all sensors that can cover a target $q_{k}$. Note that the authors in [15] assumed that $b_{i}=1$ for all sensors. The above formulation is next relaxed to an Linear Programming (LP). The authors proposed an heuristic to solve this formulation. Since the running time is $O\left(n^{3}\right)$ to solve the LP if using Ye's algorithm [8], the time complexity for the LP based algorithm in [15] is $O\left(p^{3} n^{3}\right)$, which is quite high. For the practical reasons, the authors in [15] also proposed another heuristic to find a family of ordered pairs $\left(S_{j}, t_{j}\right)$. This heuristic constructs the set covers recursively. For each set cover, the heuristic identifies a critical target, i.e., the target most sparsely covered, both in terms of number of sensors as well as with regard to the residual energy of those sensors. After selecting the critical target, the heuristic selects the sensors with greatest contribution with respect to the critical target. The contribution can be measured by the number of uncovered targets that sensor can cover and the remaining energy of that sensor. The time complexity of this heuristic is $O\left(d m^{2} n\right)$ where $m$ is the number of targets and $d$ is the number of sensors that covers the most sparsely covered targets. The simulation results show that the second heuristic runs much faster than the first heuristic which is a LP based algorithm.
All of these algorithms are centralized, which is impossible from the practical point of view. Thus we next present the distributed and localized algorithms.

\subsection{Distributed and Localized Algo- rithms}

In large-scale distributed systems, it is almost impossible to use the centralized algorithms. Instead, the nodes should do all the calculation and obtain the information locally and quickly. The distributed and localized algorithms satisfy this need. In distributed algorithms, the decision process is decentralized. In the distributed and localized algorithms, the decision process is not only distributed but also requires only a constant number of communication rounds.

Tian and Georganas in [19] proposed a distributed and localized algorithm to solve the area coverage problem, called Node Scheduling Scheme Based On Eligibly rule (SBO). In the SBO rule, the operation is divided into rounds such that at each round, the sensors decide their own status, i.e., whether to sleep or be active. At each round, the active sensors are active to cover the given area where all the other sensors are in the sleep mode. This operation repeatedly runs for next round. The main question needed to address here is that what rule the sensors should follow to determine their status. The authors proposed the Coverage-based Off-duty Eligibility rule $(\mathrm{CBO})$ to address this question. In the CBO rule, a sensor decides to turn it off when its sensing area is covered by its neighbors, called sponsors. To avoid blind point, which may happen when two or more neighboring sensors expect each other's sponsoring, a Backoff-based scheme was also introduced in [19]. This scheme lets each sensor delay the decision process with a random period of time. To obtain neighboring information, each sensor broadcasts a position advertisement message containing node ID and node location at the beginning of each round. One important factor needed to address here is what synchronization mechanism is used. However, the authors did not discuss about this prob- 
lem. There is no proof on the performance ratio of this algorithm either.

Focusing on distributed and localized solutions, Thai et al. proposed two $O(\log n)$ approximation distributed and localized algorithms for the target coverage problem [14]. The proposals organize sensors into non-disjoint set covers such that each set completely covers all the targets. Also, for each set cover $S_{j}$, the algorithms determine its active time $t_{j}$. In particular, the authors first reduce the target coverage problem to the domatic number problem in directed graphs. The domatic number is defined as the maximum number of disjoint dominating sets in a given graph. To reduce the target coverage problem to the domatic number problem, the authors proposed a distributed and localized algorithm to construct a directed graph $G^{\prime}$ from a given graph $G$. Graph $G=(X, Y ; E)$ represent a coverage relationship between sensors and targets. $X$ is a set of sensors, $Y$ is a set of targets and $E$ is a set of directed edges from $X$ to $Y$. If $u v \in E$, then sensor $u$ is able to cover target $v$. The directed graph $G^{\prime}$ holds this property: A set $S$ is a dominating set of $G^{\prime}$ if and only if $S$ completely covers all the targets in $G$.

After constructing the directed graph $G^{\prime}$, the target coverage problem on $G$ is now equivalent to the maximum number of disjoint dominating sets (MDS) on $G^{\prime}$. Note that although the solution of the MDS problem of $G^{\prime}$ is disjoint, the corresponding cover sets of $G$ are not necessary to be disjoint. To solve the MDS problem, Thai et al. proposed two distributed and localized algorithms, one for the uniform case and another for the general case. The uniform case is that the active time $b_{i}$ of each sensor in $G^{\prime}$ are equal where the general case is otherwise. In both solutions, the authors used the coloring method. The authors proved that $\frac{\left(\delta^{-}+1\right)(1-o(1))}{\ln n} \leq D\left(G^{\prime}\right)$ where $D\left(G^{\prime}\right)$ denotes the domatic number of $G^{\prime}$ and $\delta^{-}$denote the minimum in-degree of $G^{\prime}$. $o(1)$ goes to 0 as $n$ goes to $\infty$. Based on this proof, the authors argued that if a node is randomly and independently assigned a color in a certain range, we can obtain a number of domi- nating sets within a factor of $O(\log n)$. Thus in their algorithms, each node randomly chooses a color $c \in\left[1, \ldots, \frac{\delta^{\prime}-(v)+1}{3 \ln n)}\right]$. After randomly choosing their own colors, all the nodes that have the same color, i.e., in the same color class, are active for $b^{\prime}$ unit time (in the uniform case where $b_{i}=b^{\prime}$ for all nodes in $G^{\prime}$ ). The active time can be decided locally as follows. Assume that the node $v$ chooses the color $c_{v}$, then node $v$ can be active from $b^{\prime} \cdot\left(c_{v}-1\right)$ to $b^{\prime} \cdot c_{v}$. The authors in [14] also proved that their algorithms have a performance ratio within a factor of $O(\log n)$.

\section{4 k-Coverage}

In this section, we discuss the recent proposals on k-Coverage problem. As mentioned in section 2 , the objective of this problem is to find a family of ordered pairs $\left(S_{j}, t_{j}\right)$ such that the total time $t_{j}$ is maximum and each target can be monitored by at least $k$ distinct sensors in each set $S_{j}$. Not many work in recent research literature have addressed this problem.

The work in [15] can be extended to solve this problem. Recall that the authors in [15] formulated the target coverage problem as follows:

$$
\begin{array}{ll}
\text { maximize } & \sum_{j=1}^{p} t_{j} \\
\text { subject to } & \sum_{j=1}^{p} x_{i j} t_{j} \leq 1 \forall i \\
& \sum_{i \in C_{k}} x_{i j} \geq 1 \forall k, j \\
\text { where } & x_{i j}=0,1\left(x_{i j}=1 \text { iff } s_{i} \in S_{j}\right)
\end{array}
$$

Note that the constraint $\sum_{i \in C_{k}} x_{i j} \geq 1$ is to guarantee that each target is monitored by at least one sensor. If we change 1 to $k$, that is, $\sum_{i \in C_{k}} x_{i j} \geq k$, then we have each target being monitored by at least $k$ distinct sensors. Hence, using the same algorithms proposed in [15], the k-Coverage problem can be solved.

In [21], the authors showed an important observation. First, let us introduce a necessary definition.

Definition 12 Intersection Point: Given a coverage region $A$, a point $q$ is called an intersection point if: 
1. If $q \in A, q$ is an intersection point of the sensing cycles of any two sensors $u$ and $v$

2. If $q$ is on A's boundary, $q$ is an intersection point of sensor $v$ and $A$ if $|p v|=r_{v}$

Recall that $r_{v}$ is the sensing range of sensor $v$. Wang et al. [21] proved the following theorem:

Theorem 2 Given a convex region $A$ and a set of sensors $S, A$ is $k$-covered if 1) A contains intersection points and 2) all intersection points are $k$-covered.

Based on this theorem, the authors in [21] proposed an algorithm called Coverage Configuration Protocol (CCP) for the k-coverage problem. Here, the authors defined the problem slightly different to our definition. Instead of global lifetime optimization, the authors tried to find a maximum number of sensors that can be off. This is a local optimization. CPP is a distributed and localized algorithm. This algorithm can dynamically configure the network to provide different coverage degrees $k$ requested by applications without incurring high communication overhead. From the above theorem, a sensor can turn off if all the intersection points inside its sensing circle are at least $k$-covered. Thus the central part of the CPP is to determine the coverage of all intersection points in the same region. To find all the intersection points, each sensor maintains a table with neighbors information: location and status. Each sensor also periodically broadcasts a HELLO message with its current location and status so the neighbors can update their tables. All sensors start in the SLEEP state for a random time. When a sensor wakes up, it enters into a LISTEN mode. Here, the coverage degrees of all intersection points are calculated to determine the sensor's status: to be ACTIVE or go back to SLEEP. When a sensor is ACTIVE, every time it receives a HELLO message, it will re-evaluate the coverage eligibility.

\section{Connected Coverage and Connected k-Coverage}

One simple yet important theorem about the relationship between coverage and connectivity was addressed by Zhang and Hou [11] as follows:

Theorem 3 When the number of sensors in any finite area is finite, a necessary and sufficient condition for the complete coverage of a convex region to imply connectivity is $R \geq 2 r$.

Recall that $R$ is a communication range and $r$ is a sensing range of a sensor. This theorem indicates that all the above algorithms on the area coverage problem are also a solution for the connected coverage problem.

In [11], Zhang and Hou further discussed the case $R \geq r$ and proposed a distributed and localized algorithm called Optimal Geographical Density Control (OGDC). Similar to [21], the authors in [11] presented an interesting fact. An area is completely covered if there are at least two sensing ranges that intersect and all intersection points are covered. The algorithm runs in rounds, and at each round, the sensors can decide in status, i.e., $\mathrm{ON}$ and $\mathrm{OFF}$, based on the following scheme. Initially, all sensors are in the UNDECIDED states. A set of one or more starting sensors are selected as working sensors. A starting sensor broadcasts a power-on message as well as changes its state to ON. When a sensor receives a power-on message, it checks whether its neighbors cover its sensing area. If yes, it changes its state to OFF. At the end of each round, all UNDECIDED sensors will change to either ON or OFF state.

Wang et al. in [21] generalized the above theorem to address the communication and connectivity relationship on the $k$-coverage problem.

Theorem 4 Given a convex region $A$, a set of sensors that $k$-covers $A$ forms a $k$-connected communication graph if $R \geq 2 r$.

A k-connected graph is a graph such that removing any $k-1$ vertices still results in a connected graph. This theorem indicates that all 
the proposals on $k$-coverage problem are also the solutions for the connected $k$-coverage problem when $R \geq 2 r$. When $R<2 r$, Wang et al. proposed an algorithm which is an integration of the CCP protocol (mentioned in the previous section) and SPAN [27]. This integration provides a 1 -connected $k$-coverage network. SPAN is a distributed algorithm that conserves energy by putting the unnecessary sensors into a sleep mode while maintaining connectivity. This characteristic of this protocol results in a 1-connected for the integration protocol. The integration protocol is similar to CPP, except the eligibility rule, which is updated as follows: 1) A sensor is active if it satisfies both the eligibility rules of CPP and SPAN, 2)A sensor goes to the sleep mode if it satisfies neither the CPP eligibility rule nor the SPAN eligibility rule.

In [22], Zhou et al. proposed a greedy algorithm for the connected $k$-coverage problem. This greedy algorithm is centralized with an approximation ratio within a factor of $O(\lg n)$. They also proposed a distributed version of this greedy algorithm. In their work, instead of focusing on the global optimization, they addressed the local optimization, that is to find a connected $k$-cover of smallest size. To find this set, at each iteration, a set of sensors $S$ is added to a current chosen set $M$ based on these criteria: 1) A set $S$ forms a communication path to a sensor $s_{l} \in M$, 2) $S$ has a most $k$-benefit, i.e., covers the most uncovered fields.

All of these work on connected coverage problem focus on the area coverage. No work has addressed this problem on the target coverage yet and we expect to see more research in this specific area.

\section{Minimum Coverage Breach}

Although prolonging network lifetime for the coverage problem in wireless sensor networks has been studied extensively, there is not much research on the consideration of transmission bandwidth constraints when organizing sensors into set covers. The first work that introduced this problem is [9]. Cheng et al. in [9] modeled the Minimum Breach Problem as an Integer Programming in which sensors are organized into disjoint set covers where the size of each set is less than or equal to $W$ and the overall breach is minimized. The number of these sets is set to $\frac{n}{W}$ where $n$ is the number of sensors. In case $n$ is not divisible by $W$, ceiling or floor are used. The authors formulated this problem as follows:

$$
\begin{array}{ll}
\operatorname{minimize} & \sum_{j=1}^{p} \sum_{k=1}^{m}\left(1-y_{j k}\right) \\
\text { subject to } & \sum_{j=1}^{p} x_{i j}=1 \forall i \\
& \sum_{i=1}^{n} a_{i j} x_{i j} \geq y_{j k} \forall j, k \\
& \sum_{i=1}^{n} x_{i j}=W \forall j \\
\text { where } & x_{i j}=0,1\left(x_{i j}=1 \text { iff } s_{i} \in S_{j}\right) \\
& y_{j k}=0,1\left(y_{j k}=1 \text { iff } S_{j} \text { covers target } q_{k}\right)
\end{array}
$$

The authors then presented two centralized heuristics called RELAXATION and MINBREACH. The RELAXATION is a linear programming based algorithm. Since the running time of the LP is $O\left(n^{3}\right)$, Cheng et al. introduced the second heuristic called MINBREACH to avoid solving the LP. The second heuristic is based on the matrix techniques and operations. Note that the disjoint constraint is not necessary to obtain a maximum network lifetime nor to obtain a minimum coverage breach.

In [10], Thai et al. proposed two centralized algorithms for the Minimum Coverage Breach. The main approach is to organize sensors into a number of non-disjoint set covers. The first algorithm is to find a family of ordered pairs $\left(S_{j}, t_{j}\right)$ to maximize network lifetime while making sure that the number of sensors in each set cover $S_{j}$ is less than or equal to $W$ and no coverage breach is allowed. In other words, each set $S_{j}$ completely covers all targets. This algorithm is an extended version of the algorithm proposed in [15] by adding one more constraint $\left(\sum_{i=1}^{n} x_{i j} \leq W\right)$ into the LP formulation:

$$
\begin{array}{ll}
\text { maximize } & \sum_{j=1}^{p} t_{j} \\
\text { subject to } & \sum_{j=1}^{p} x_{i j} t_{j} \leq 1 \forall i \\
& \sum_{i \in C_{k}} x_{i j} \geq 1 \forall k, j \\
& \sum_{i=1}^{n} x_{i j} \leq W \forall j \\
\text { where } & x_{i j}=0,1\left(x_{i j}=1 \text { iff } s_{i} \in S_{j}\right) \\
& 0 \leq t_{j} \leq 1
\end{array}
$$


The second algorithm allows coverage breach occurred. The objectives of this algorithm are to maximize network lifetime as well as to minimize the coverage breach. The authors in [10] formulated this joint optimization problem as follows:

$$
\begin{array}{ll}
\operatorname{minimize} & \sum_{j=1}^{p} \sum_{k=1}^{m}\left(t_{j}-t_{j} \cdot y_{j k}\right) \\
\text { subject to } & \sum_{j=1}^{p} x_{i j} t_{j} \leq 1 \forall i \\
& \sum_{i \in C_{k}} x_{i j} \geq y_{j k} \forall k, j \\
& \sum_{i=1}^{n} x_{i j} \leq W \forall j \\
\text { where } & x_{i j}=0,1\left(x_{i j}=1 \text { iff } s_{i} \in S_{j}\right) \\
& y_{j k}=0,1\left(y_{j k}=1 \text { iff } S_{j} \text { covers } q_{k}\right) \\
& 0 \leq t_{j} \leq 1
\end{array}
$$

Using the relaxation technique, the authors transformed this formulation to the linear programming. The authors then proposed an approximation algorithm to find a number of sets $S_{j}$ and their active time $t_{j}$. By allowing sensors to be divided into non-disjoint sets, the results obtained from this algorithm is about $45 \%$ less than that in [9].

\section{Discussion and Outlooks}

In this paper, we present the recent proposals in research literature on the coverage problem. The main objective of the coverage problem is to maximize network lifetime due to the power scarcity. Most of the proposals use the scheduling mechanism, that is to organize sensor into a number of subset such that each set completely covers an interest region. These sets can be active successively to prolong network lifetime. When one set is active to do the sensing task, the other sets are in the sleep mode.

Besides the objective of maximizing network lifetime, many work also considered other network constraints, such as connectivity, bandwidth constraints, and fault tolerance. One important observation is that if the communication range is at least twice the sensing range, the complete coverage of a convex region implies the connectivity.

There is another type of the general coverage problem called barrier coverage which is not in the scope of this paper. In this problem, the objective is to minimize the probability of undetected penetration through the barrier. The maximal breach path and the maximal support path model was introduced by Meguerdichian et al. [23]. Another model, called exposure-based model, was also introduced by Meguerdichian et al. [24]. Readers who are interested in this topic can refer to these papers [23]-[26].

Although there are many work on the coverage problem, there are still many unaddressed challenges. Most of proposals are centralized algorithms which are not practical. Even though they are centralized, most of the authors cannot prove the approximation ratio of their algorithms. In addition, from the practical point of view, more distributed and localized algorithms need to study. A few localized algorithms have been proposed but due to the nature of the proposals, it is hard to prove their performance ratio.

The relationship between coverage and connectivity is true for a convex area, but not necessary true for a set of targets. None of these work has studied this relationship in the target coverage yet. Hence more work on the connected target coverage problem need to be done.

\section{References}

[1] D. Estrin, R. Govindan, J. Heidemann, and S. Kumar, Next Century Challenges: Scalable Coordination in Sensor Networks, in Proceeding of ACM International Conference on Mobile Computing and Networking (MOBICOM 99), Washington 1999

[2] J. Kahn, R. Katz, and K. Pister, Nexst Century Challenges: Mobile Networking for Smart Dust, in Proceeding of ACM International Conference on Mobile Computing and Networking (MOBICOM 99), Washington 1999

[3] A. Cerpa, J. Elson, D. Estrin, L. Girod, M. Hamilton, and J. Zhao, Habitat Monitoring: Application Driver for Wireless Communications Technology, in Proceeding of ACM SIG- 
COMM Workshop on Data Communications, Latin America and the Caribbean Costa Rica 2001

[4] I.F. Akyildiz, W. Su, Y. Sankarasubramaniam, and E. Cayirci, A Survey on Sensor Networks, IEEE Communications Magazine, (Aug 2002), 102-114

[5] L. Benini, G. Castelli, A. Macii, E. Macii, M. Poncino, and R. Scarsi, A Discrete-Time Battery Model for High-Level Power Estimation, Proceedings of DATE, 35-39, 2000

[6] V. Raghunathan, C. Schurgers, S. Park, and M..B. Srivastava, Energy-Aware Wireless Microsensor Networks, IEEE Signal Processing Magazine , 40-50, 2002

[7] N. Garg and J. Konemann, Faster and Simpler Algorithms for Multicommodity Flow and Other Fractional Packing Problems, Proc. of FOCS, 1997

[8] Y. Ye, An $O\left(n^{3} l\right)$ Potential Reduction Algorithm for Linear Programming, Mathematical Programming, Vol 50, pp 239-258, 1991

[9] M. X.. Cheng, L. Ruan, and W. Wu, Achieving Minimum Coverage Breach under Bandwidth Constraints in Wireless Sensor Networks, Proceedings of the 24th conference of the IEEE Communications Society (INFOCOM), 2005

[10] M. T. Thai, Y. Li, F. Wang, and D.-Z. Du, Minimum Coverage Breach and Maximum Network Lifetime in Wireless Sensor Networks, Manuscript

[11] H. Zhang and J.C. Hou, Maintaining Sensing Coverage and Connectivity in Large Sensor Networks, The Wireless Ad Hoc and Sensor Networks: An International Journal, Jan 2005

[12] H. Gupta, S. R. Das and Q. Gu, Connected Sensor Cover: Self-organization of
Sensor Networks for Efficient Query Execution, MobiHoc, Annapolis, Maryland, USA, June 2003.

[13] P. Berman, G. Calinescu, C. Shah, and A. Zelikovsky, Power Efficient Monitoring Management in Sensor Networks, Proceedings of WCNC'04, March 2004

[14] M. T. Thai, Y. Li, F. Wang, and D.-Z. Du, $O(\log n)$ Distributed Algorithms on the Coverage Problem in Wireless Sensor Networks, manuscript

[15] M. Cardei, M. T. Thai, Y. Li, and W. Wu, Energy-Efficient Target Coverage in Wireless Sensor Networks, Proceedings of the 24th conference of the IEEE Communications Society (INFOCOM), 2005

[16] M. Cardei and D.-Z. Du, Improving Wireless Sensor Network Lifetime through Power Aware Organization, ACM Wireless Networks, Vol. 11, No. 3, 2005.

[17] S. Slijepcevic and M. Potkonjak, Power Efficient Organization of Wireless Sensor Networks, IEEE International Conference on Communications, June 2001

[18] M. Cardei, D. MacCallum, X. Cheng, M. Min, X. Jia, D. Li, and D.-Z. Du, Wireless Sensor Networks with Energy Efficient Organization, J. of Interconnection Networks, vol 3, No 3-4, pp 213-229, 2002

[19] D. Tian and N. D. Georganas, A CoveragePreserving Node Scheduling Scheme for Large Wireless Sensor Networks, in Proceeding of the 1st ACM Workshop on Wireless Sensor Networks and Applications, 2002

[20] F. Ye, G. Zhong, Z. Lu, and L. Zhang, Energy Efficient Robust Sensing Coverage in Large Sensor Networks, UCLA Techical Report, 2002

[21] X. Wang, G. Xing, Y. Zhang, C. Lu, R. Pless and C. D. Gill, Integrated Coverage and 
Connectivity Configuration in Wireless Sensor Networks, 1st ACM Conference on Embedded Networked Sensor Systems, 2003.

[22] Z. Zhou, S. Das, and H. Gupta, Connected $\mathrm{K}$-Coverage Problem in Sensor Networks, $I C$ $C C N, 2004$

[23] S. Meguerdichian, F. Koushanfar, M. Potkonjak, and M. Srivastava, Coverage Problem in Wirelss Ad-Hoc Sensor Networks, IEEE INFOCOM, 2001

[24] S. Meguerdichian, F. Koushanfar, G. Qu, and M. Potkonjak, Exposure in Wireless AdHoc Sensor Networks, MOBICOM, 2001

[25] X.-Y. Li, P.-J. Wang, and O. Frieder, Coverage in Wirelss Ad-Hoc Sensor Netwroks, IEEE Transactions on Computers, Vol 52, pp 753-763, 2002

[26] B. Liu and D. Towsley, On the Coverage and Detectability of Wireless Sensor Networks, WiOpt: Modeling and Optimization in Mobile, Ad Hoc and Wireless Networks, 2003

[27] B. Chen, K. Jamieson, H. Balakrishnan, and R. Morris, SPAN: An EnergyEfficient Coordination Algorithm for Topology Maintenace in Ad hoc Wirelss Networks, ACM/IEEE International Conference on Mobile Computing and Network, 2001 